\title{
Numerical Studies of Phase Separation in Models of Binary Alloys and Polymer Blends
}

\author{
James D. Gunton, Raul Toral* and Amitabha Chakrabarti ${ }^{*}$ \\ Physics Department and Center for Polymer Science and Engineering Lehigh University, Bethlehem, PA 18015, U.S.A.
}

Received October 30, 1989; accepted November 13,1989

\begin{abstract}
We review some recent results concerning numerical studies of the CahnHilliard equation in three dimensions to describe the phase separation process in a mixture after a quench within the coexistence curve. We focus on the late time behavior for two experimentally relevant systems: binary alloys and binary polymer blends. We find that, in both systems, dynamical scaling with a time dependent characteristic length $R(t)$ holds at sufficiently late times and that the late-time behavior for $R(t)$ can be described by a modified Lifschitz-Slyozov law: $R(t)=c+d t^{n}$, where $n=1 / 3$. For polymer mixtures, the independence of the growth law exponent $n$ of the quench temperature is in contradiction with some recent experiments on polymer systems.
\end{abstract}

\section{Introduction}

The process of phase separation is the subject of many theoretical and experimental investigations in the field of small molecule or atomic systems, such as binary alloys, fluid mixtures and inorganic glasses [1], as well as in the field of polymer mixtures [2-4]. When such a system is quenched from a homogeneous, high temperature, phase to a point deep inside the coexistence curve (a critical quench), a small amplitude, long wavelength instability develops. This phenomenon is known as spinodal decomposition. At later times, the small inhomogeneities in the order parameter evolve into macroscopic domains of one or the other phase and an interconnected structure is formed. The theoretical understanding of this phase separation process is based mainly on the Cahn-Hilliard-Cook (CHC) [5] formulation. The main ingredient of the $\mathrm{CHC}$ theory is a conserved field variable $\phi(x, \tau)$ representing the local concentration of one of the components of the mixture (or, sometimes, the difference between the local concentration of the two components of a binary mixture). The time variation of this field is related to the functional derivative of a coarse-grained free-energy functional $F[\phi]$ plus a thermal noise $\eta(x, \tau)$ in the following way:

$\frac{\partial \phi}{\partial \tau}=M \nabla^{2} \frac{\delta F}{\delta \phi}+\eta(x, \tau)$

where $M$ is the mobility (assumed constant in the theory) and the thermal noise $\eta$ satisfies the fluctuation-dissipation theorem:

$\left\langle\eta(x, \tau) \eta\left(x^{\prime}, \tau^{\prime}\right)\right\rangle=-2 k_{\mathrm{B}} T M \nabla^{2} \delta\left(x-x^{\prime}\right) \delta\left(\tau-\tau^{\prime}\right)$

\footnotetext{
* Permanent address: Department de Fisica, Universitat de las Illes Balears, E-0707l Palma de Mallorca, Spain.

$\ddagger$ Present address: Department of Physics, Kansas State University, Manhattan, KS 66506, U.S.A.
}

in order to ensure approach to equilibrium at a temperature $T$. The details of the free-energy functional $F[\phi]$ depend on the particular system one wishes to consider. Analytical studies of this field-theoretical model have been reasonably successful in describing the early time regime of the phase separation process [6-8] but, due to their approximate nature, they are not as useful in the so-called late stages. This late time regime is characterized by the coarsening of domains separated by interfaces whose thickness is proportional to the equilibrium correlation length and, thus, much smaller than the characteristic domain size. The well-known Lifshitz-Slyozov [11] theory, which assumes a limitingly small volume fraction of one of the two phases, is not strictly applicable to the case of a critical quench. Attempts have been made to use renormalization group [9] and low-temperature perturbation methods [10] in order to study the late-stages of evolution process for a critical quench but the results of these studies are somewhat controversial. It seems then that numerical simulation would play a useful role, at least for the present time, in our ability to understand and to predict the late time behavior of such a complicated system.

Numerical simulations for binary alloy systems traditionally have focussed mainly on discrete models [12] (Ising type) with a microscopic dynamics of spin exchange (Kawasaki dynamics) which conserves the order parameter. Recent numerical studies, however, of both the Ising model $[13,14]$ and the CHC model in two dimensions [14-16] (and an alternative cell-dynamics version [17-19] of the CHC model) have suggested that the time dependent behavior of the characteristic size of the domains (as characterized by a power law behavior in time) and the scaling function derived from the pair-correlation function are the same within the numerical accuracy of the studies [19]. For many years, the only numerical results available in three dimensions were those from earlier pioneering Monte Carlo simulation of Kawasaki dynamics in the Ising model [12]. Recently, useful new results have been obtained by us $[20,21]$ for the three dimensional $\mathrm{CHC}$ model, which we will present in this review article.

Analytical studies of spinodal decomposition in polymer mixtures have been carried out by several authors [22] by using the $\mathrm{CHC}$ formulation with a suitable free energy functional (based on the Flory-Huggins-de Gennes free energy). Due to the same problems one faces in the study of binary alloys, the calculations in polymer systems have dealt mainly with the early stages of the phase separation process. It seems, again, that numerical simulations can successfully probe the late time predictions of the theory that are unaccessible to analytical calculations. Monte Carlo simulation techniques have been used to study early stages of phase separation in a 
lattice model of polymer mixtures in two and three dimensions $[23,24]$. Here, we discuss results of a detailed numerical study of the $\mathrm{CHC}$ model in three dimensions starting from a full Flory-Huggins-de Gennes type free energy and numerically integrating the time evolution equation of the conserved order parameter.

The main results of these studies are that the domain growth law for binary alloys is described by the modified Lifshitz-Slyozov law proposed by Huse [26]. In addition, the pair correlation function and the structure factor are shown to exhibit dynamical scaling for the binary alloy system. For polymer blends we find that the growth law for the domain size is independent of the final temperature of quench. As well, dynamical scaling is satisfied both for the pair correlation and the structure factor at sufficiently late times.

\section{Phase separation in binary alloys}

\subsection{Model and numerical methods}

In this section we focus on computer simulation studies of a three-dimensional model system corresponding to the case of quenched binary alloys. The functional $F[\phi]$ for binary alloy systems is usually assumed to have the Ginsburg-Landau form:

$$
F=\frac{1}{2} \int \mathrm{d} x\left[-b \phi^{2}+\frac{u}{2} \phi^{4}+K|\nabla \phi|^{2}\right]
$$

where $b, u$, and $K$ are phenomenological positive parameters. The resulting equation of motion obtained after substituting eq. (3) into eq. (1) is:

$\frac{\partial \phi}{\partial \tau}=M \nabla^{2}\left(-b \phi^{2}+u \phi^{3}-K \nabla^{2} \phi\right)+\eta$

Following Grant et al. [8] this equation can be written in a simpler fashion by suitable rescaling of the field, the distances and the time. The resulting dimensionless equation is:

$\frac{\partial \psi}{\partial t}=\frac{1}{2} \nabla^{2}\left(-\psi^{2}+\psi^{3}-\nabla^{2} \psi\right)+\sqrt{ } \varepsilon v$

where $\psi=\phi /(b / u)^{1 / 2}, r=x /(K / b)^{1 / 2}, t=\tau /\left(K / 2 M b^{2}\right), \varepsilon=$ $\left(k_{\mathrm{B}} T u / b^{2}\right) /(b / K)^{\mathrm{d} / 2} \cdot v(r, t)$ is a Gaussianly distributed stochastic variable of mean zero and correlations given by $\left\langle v(r, t) v\left(r^{\prime}\right.\right.$, $\left.\left.t^{\prime}\right)\right\rangle=-\nabla^{2} \delta\left(r=r^{\prime}\right) \delta\left(t-t^{\prime}\right)$. At low temperatures, the role of the noise term is thought to be very small and does not affect some important features of the late stages of the evolution (e.g., neither the growth law for the characteristic domain size nor the scaling functions), as pointed out in the studies of the two dimensional version of the model [16]. Equation (5) in the absence of the noise term $(\varepsilon=0)$ is called the Cahn-Hilliard equation and is the subject of several numerical studies.

Numerically solving eq. (5) in three dimensions is computationally a very demanding task, even in the absence of the noise term. We have used a finite difference scheme for both the spatial and temporal derivitives. The spatial discretization is achieved by replacing the continuous space of position vectors $r \equiv(x, y, z)$ by a simple cubic lattice with $N=L^{3}$ sites and lattice spacing $\delta r$ (periodic boundary conditions are assumed in order to avoid surface effects). We integrate numerically eq. (5) by using a first order Euler scheme:

$$
\psi(r, t+\Delta t)=\psi(r, t)+\Delta t \frac{\partial \psi}{\partial t}
$$

In order to carry out the calculations within a reasonable amount of computer time one would like to choose a large time step and a moderately large system size. However, the discretized version of eq. (5) develops a subharmonic bifurcation kind of instability for large time steps [16]. A linear stability analysis in three dimensions [21] shows that this bifurcation can be avoided by choosing the parameters $\Delta t$ and $\delta r$ such that the following inequality is satisfied:

$$
\Delta t<\frac{(\delta r)^{4}}{36-3(\delta r)^{2}}
$$

This simple criterion turns out to be a necessary but not sufficient condition for the stability of the numerical integration. We have chosen in our simulation $\delta r=1.7$ and $\Delta t=$ 0.1 . This value of $\Delta t$ is more than three times smaller than the "safe" value predicted by eq. (7). We have found that smaller values of $\Delta t$ do not change quantities that express a global behavior, such as the structure function, the pair correlation function or the typical domain size. We have considered a simple cubic lattice with $L=66$, which is equivalent to saying that the linear dimension of the system (in the dimensionless units described before) $L_{\mathrm{a}} \approx 112$ units. We choose the initial field configurations to be distributed between -0.125 and 0.125 , with the order parameter equal to zero (i.e., a critical quench). In order to average over the initial random configurations we have performed 46 runs out to $t=10000$ and 25 runs out to $t=20000$.

\subsection{Computation of different probes}

Dynamical scaling and growth laws for the average domain size have been traditionally analyzed in terms of the structure function:

$S(k, \mathfrak{t})=\left\langle\frac{1}{N} \sum_{r} \sum_{r^{\prime}} \mathrm{e}^{\mathrm{i} k r}\left[\psi\left(r+r^{\prime}, t\right) \psi\left(r^{\prime}, t\right)-\langle\psi\rangle^{2}\right]\right\rangle$

where the sum over the lattice and the $k$ vectors belong to the first Brillouin zone in the reciprocal space i.e.,

$k=\frac{2 \pi}{L \Delta r} \mu, \quad \mu=\left(\mu_{x}, \mu_{y}, \mu_{z}\right), \quad 0 \leqslant \mu_{x}, \mu_{y}, \mu_{z} \leqslant L-1$.

We then define a spherically averaged structure factor as:

$S(k, t)=\sum_{k-(\Delta k / 2)<|k| \leqslant k+(\Delta k / 2)} S(k, t) / n(k, \Delta k)$

where

$n(k, \Delta k)=\sum_{k-\langle\Delta k / 2)<|k| \leqslant k+(\Delta k / 2)} 1$

The quantity defined in eq. (10) denotes the number of lattice points in a spherical shell of width $\Delta k$ centered around $k$. Ideally, one should take $\Delta k$ as small as possible. A convenient value for the discretized Brillouin zone considered in the simulation, though, is $\Delta k=(2 \pi) /(L \Delta r)$. For typical measures of domain size, one usually considers either the location $k_{\mathrm{m}}(t)$ of the peak of the spherically averaged structure factor or the moment $k_{\mathrm{a}}(t)$ defined as:

$k_{\mathrm{a}}(t)=\sum_{k} k S(k, t) / \sum_{k} S(k, t)$ 
In recent years, scaling growth laws have also been analyzed in terms of the real-space pair correlation function $G(r, t)$ defined as:

$$
G(r, t)=\sum_{k} \mathrm{e}^{\mathrm{i} k r} S(k, t)
$$

In this case also we perform a spherical average procedure as described above and obtain the corresponding correlation function $G(r, t)$. However, this procedure has the disadvantage that a simple point $r$ represents an average over a spherical shell of width $\Delta r$, introducing an uncertainty in the coordinate $r$ of the order of $\Delta r / 2$. As a different measure we also study the correlation function $G_{\mathrm{a}}(x, t)$ averaged along the lattice axes:

$$
\begin{aligned}
G_{\mathrm{a}}(x, t)= & \frac{1}{3}[G(x, 0,0), t)+G(r=(0, x, 0), t) \\
& +G(r=(0,0, x), t)]
\end{aligned}
$$

The domain morphology for the conserved order parameter produces a damped oscillatory behavior in both $G(r, t)$ and $G_{\mathrm{a}}(x, t)$. This allows one to give a quantitative measure of the domain size as the location of the first zero of the correlation function, The length $R_{\mathrm{g}}\left(R_{\mathrm{a}}\right)$ was calculated fitting the four points in $G(r, t)\left(G_{\mathrm{a}}(x, t)\right)$ closest to its first zero (of which two fall on each side of its first zero) to a cubic polynomial of $r(x)$ and defining $R_{\mathrm{g}}\left(R_{\mathrm{a}}\right.$ as the value of $r(x)$ where this fitted function vanishes.

\subsection{Results and discussion}

2.3.1. Late time configuration of the system. The characteristic interconnected structure of a system at a late stage of spinodal decomposition is clearly visible in Fig. 1. In this figure positive (negative) values of the field $\psi$ are presented by opaque (transparent) points. We note that both phases form complementary spanning structures, which is similar to that observed in experiments on binary alloys [27].

The probability distribution for the field $\psi(r, t)$ is plotted in Fig. 2 for two late times during the evolution. We note that the distribution is very sharply peaked around the equilibrium values of $\psi$, i.e., -1 and +1 . This indicates that the field is in local equilibrium spatially except at the interfaces. The

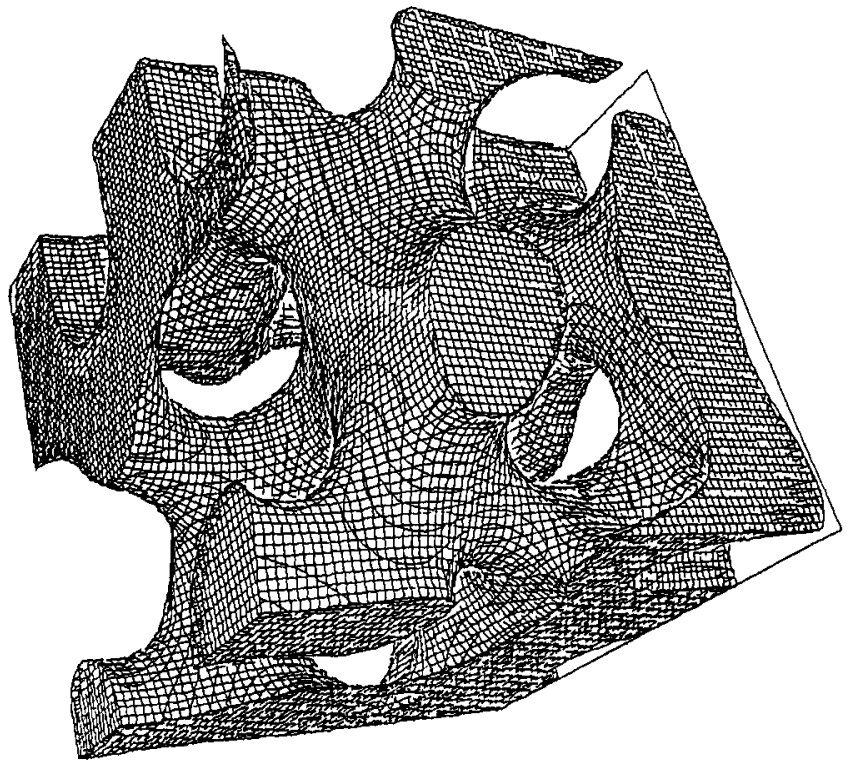

Fig. 1. Snapshot of the system configuration at $t=20000$. The region with a positive value for the concentration field is indicated opaque, while the region with a negative value is transparent. The spanning structure characteristic of spinodal decomposition is clearly visible.

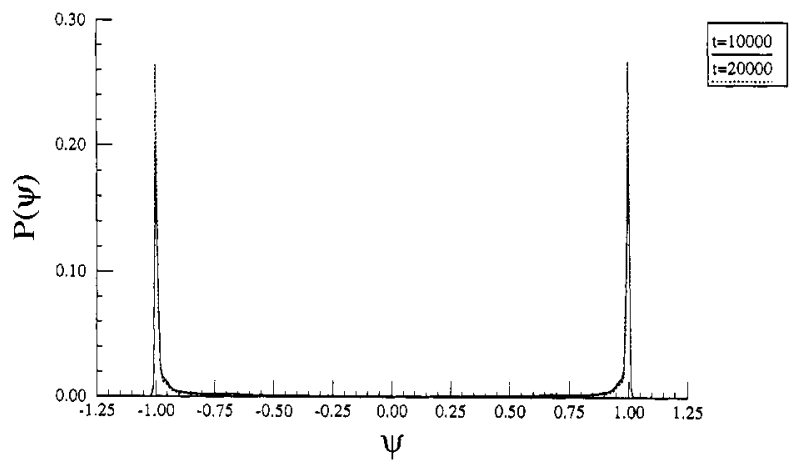

Fig. 2. Probability distribution $P(\psi(r, t))$ at two advanced times during the system evolution. Note that the distribution for both times is sharply peaked around the equilibrium values for the field, +1 and -1 .

probability distribution function $P(\psi(r, t))$ contains information about the interfaces [28] and a simple heuristic argument allows us to relate $P(\psi(t, t))$ to the interface profile. We find that the interface profile is essentially independent of time and well approximated by a hyperbolic tangent fit. We also note that the width of the interface is of the order of 1.0 and thus is about $4 \%$ of the maximum domain size. Since this number is small we believe that the system studied here is in an advanced stage of the evolution process.

2.3.2 Scaling. The late stages of the dynamical process can be described in terms of an asymptotic scaling with a time dependent length. The fundamental assumption is that in the asymptotic scaling regime one length $R(t)$ is relevant. This length $R(t)$ represents the characteristic size of the domains. Then the dynamical scaling hypothesis states that:

$G(r, t)=g(r / R(t))$
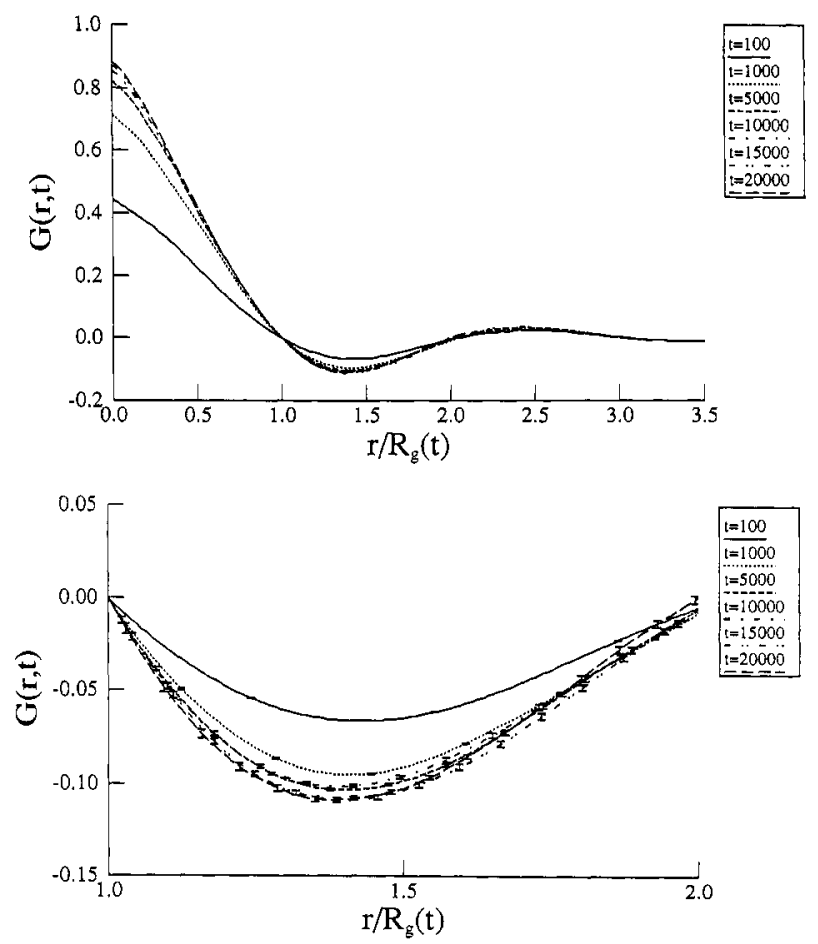

Fig. 3. Spherically averaged pair correlation function as a function of $r / R_{\mathrm{g}}$ to check scaling ansatz eq. (14). $R_{\mathrm{g}}$ is defined such that this plot goes through the point $(1,0)$. Scaling holds reasonably well after $t=5000$ although up to $t=15000$ (see Fig. 3(b)). (b) Details of Fig. 3(a) near the first minimum of the scaling function. 
and, consequently:

$S(k, t)=R(t)^{d} F(k R(t))$

where both $g(\rho)$ and $F(\chi)$ are time independent functions.

Figure 3(a) shows the scaling behavior of $G(r, t)$ with $R_{g}$ as the scaling length. The scaling hypothesis seems to be extremely well satisfied, over the whole range of values of $r$, particularly at late time. On the other hand, a detailed analysis of the first minimum of $G(r, t)$ shows that the position of this minimum does not change with time but that the value of the minimum decreases systematically, at least up to $t=15000$, see Fig. 3(b). Also, the value of the scaling function in Fig. 3(a) at the origin is increasing slowly with time. This indicates that for very small values of the scaling variable $\rho$, the curve in Fig. 3(a) is not quite yet the true scaling function. Scaling can not be checked for arbitrary large values of the scaling variable $\rho$ either, due to the finiteness of the system. The periodic boundary conditions impose a maximum distance on the lattice equal to $L_{\mathrm{a}} / 2=56$. When $t=20000, R_{\mathrm{g}}=26.4$ and the maximum value of the scaling variable $\rho$ for which we can study scaling at this late time is then $\rho=2.12$. We are, however, including in Fig. 3(a) data up to $\rho=3.5$ in order to show that scaling is very well satisfied for larger values of $\rho$ at relatively earlier times.

We also concentrated on the correlation function $G_{\mathrm{a}}(x, t)$ defined in eq. (13), mainly because there is no uncertainty in the coordinate $x$ for this function in contrast to the case of $G(r, t)$. We show the scaling of $G_{a}(x, t)$ by plotting this function against $\rho=x / R_{\mathrm{a}}$ in Fig. 4. It is not surprising that there are large error bars associated with $G_{\mathrm{a}}(x, t)$ for large values of $x$ since when $x$ is larger than $R_{\mathrm{a}}$ (which corresponds roughly to the linear size of the domains) the value of the correlation function is small and fluctuates widly. Unlesss there are a large numbers of pairs contributing to the correlation function errors become important and are actually comparable to the value of the function itself. We note that for large values of $x$, the number of pairs contributing to $G_{\mathrm{a}}(x, t)$ is much smaller than the corresponding number to $G(r, t)$ and hence $G_{\mathrm{a}}(x, t)$ shows much larger errors than $G(r, t)$ for these large values of $x$. However, when $x \approx R_{\mathrm{a}}$, the correlations are computed for pairs mainly inside bulk domains and the fluctuations in the values of the correlation function are small. Thus the length $R_{\mathrm{a}}$ calculated from the first zero of $G_{\mathrm{a}}(x, t)$ contains small errors, since $G_{\mathrm{a}}(x, t)$ itself contains small error bars in this range of $x$ values, as evident in Fig. 4. From this figure we conclude that very good scaling

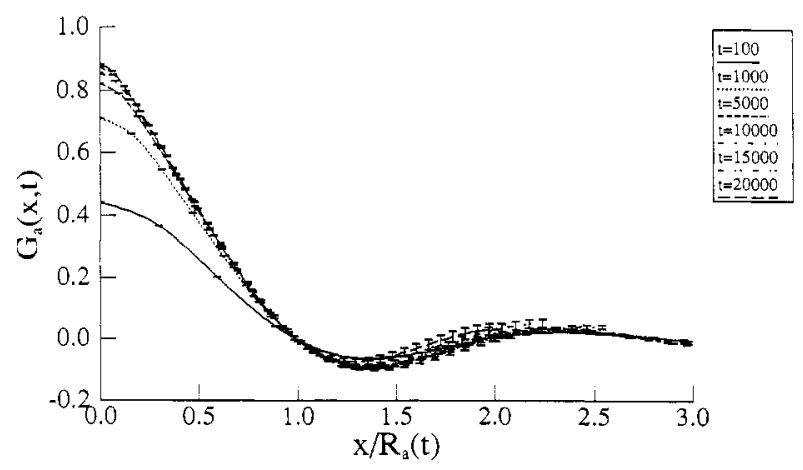

Fig. 4. Plot of the pair correlation function averaged along the lattice axes vs. $x / R_{\mathrm{a}}$ to check scaling ansatz eq. (14) and possible anisotropy in the scaling functions. $R_{\mathrm{a}}$ is defined such that this plot goes through the point $(1,0)$. The dispersion of values for large values of the abscissa is discussed in the text.
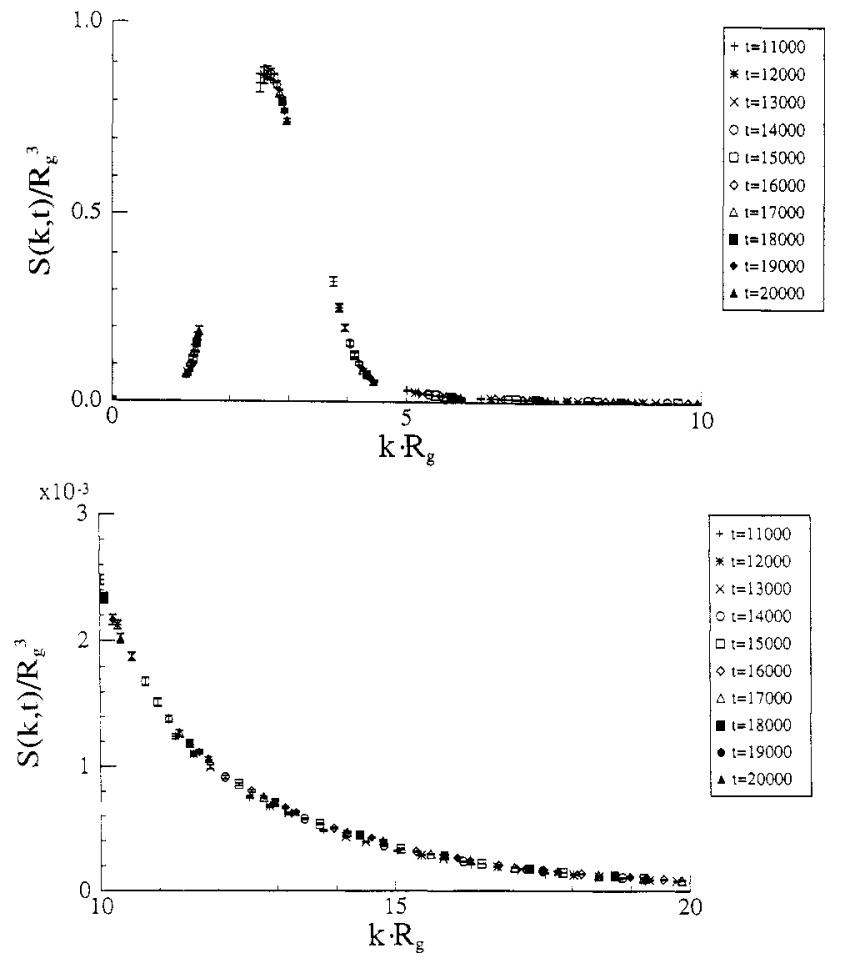

Fig. 5. Spherically averaged structure function $S(k, t)$ plotted to check scaling ansatz eq. (15) using as scaling length the first zero of the spherically averaged pair correlation. An accurate form of the scaling function for small values of the scaling variable can not be determined precisely due to the finiteness of the lattice used in the numerical study. (b) Details of Fig. 5(a): For large values of the scaling variable plotted here we can determine very precisely the predicted form of the scaling function.

holds at least for $t \geqslant 10000$ and values of $x \approx R_{\mathrm{a}}$ whereas large error bars prevent us from commenting about scaling for larger values of $x / R_{\mathrm{a}}$

The scaling ansatz, eq. (15) can be tested by plotting $S(k, t) / R_{\mathrm{g}}(t)^{3}$ vs, $\chi=k R_{\mathrm{g}}(t)$ and checking whether the resulting functions are independent of time. In Figs. 5(a) and 5(b) we show such plots for late times. It is clear from the latter figure that dynamical scaling is well satisfied for relatively large values of the scaling variable $\chi$, say $\chi \geqslant 4.0$. On the other hand, for small values of $\chi$ the lattice discretization does not leave us with enough points to make a conclusive statement about scaling. However, we believe that the asymptotic scaling function will not be significantly different from that shown in Fig. 5(a). We have compared the scaling function with previous Monte Carlo data as well as some other theoretical and phenomenological results. We find that the scaling function computed in this study is narrower around the maximum than that observed in the Monte Carlo study. Although it is difficult to compare time-scales of the two different models, due to the inherent coarse-grained nature of the model studied here, we believe that the latest time of evolution studied in the Monte Carlo simulations for the "deep" critical quenches (less than 2000 Monte Carlo steps) is simply too small to be in the scaling regime. We have suitably rescaled the axes in Fig. 5(a) in order to compare with the phenomenological expression for the scaling function introduced by Furukawa [29]. We find that this form does not agree with our data. Also, the scaling function predicted in a recent field theoretical low temperature expansion [10] is not consistent with our scaling function.

2.3.3 Growth law: The characteristic length scale given by any of the above measures, $R_{\mathrm{g}}(t), R_{\mathrm{a}}(t)$, or $k_{\mathrm{a}}(t)^{-1}$ is expected 
to behave as $t^{a}$ for sufficiently late time $t$. The classical theory of Lifshitz and Slyozov, valid only in the limit where the volume fraction occupied by droplets goes to zero, i.e., near the coexistence curve in thenucleation regime, predicts $a=$ $1 / 3$ independent of the dimension. The Lifshitz-Slyozov theory, based on a mechanism of evolution governed by bulk diffusion across the interfaces, has been qualitatively extended to the case of equal volume fraction of the two phases with the prediction [26] $a=1 / 3$. On the other hand, a recent theoretical study of the dynamics (combined with numerical simulations) predicts $a=1 / 4$ for a critical quench [10]. (A recent study proposes a crossover to $a=1 / 3$, however [30].) Experiments in various systems have been analysed in terms of effective exponents a which lie in the range $0.15-0.37$. In the Monte Carlo studies of the kinetic Ising model in three dimensions, the authors interpreted their results for the domain size in terms of an effective exponent in the range $0.19-0.35$, the smaller exponents corresponding to the critical quenches, although the authors claimed that the data are always compatible with $a=1 / 3$. Figs. 6(a)-(c) show different measures of the characteristic length plotted against $t^{1 / 3}$ and $t^{1 / 4}$. Although it is of course, difficult to distinguish between exponents $1 / 3$ and $1 / 4$ since their difference is small, the presented figures show tht $R(t)=c+d t^{1 / 3}$ is a better global fit than $R(t)=c+d t^{1 / 4}$ for all the above measures. This visual demonstration is not, certainly, the best way of extracting a growth law exponent. We also tried to find the best fit of our data to the more general expression $R(t)=c+d t^{a}$. We have found the best fit for $50 \leqslant t \leqslant 20000$ for $R_{\mathrm{g}}$ provides $a=0.32 \pm 0.02$. For $R_{\mathrm{a}}(t)$ and $k_{\mathrm{a}}(t)^{-1}$ the best fits over the same time interval give $a=0.337 \pm 0.008$ and $a=0.35 \pm 0.03$, respectively. The errors are based purely on the statistical errors of the different lengths, without taking into account the possible inherent systematic errors in $R_{\mathrm{g}}$ and $k_{\mathrm{a}}(t)^{-1}$. Another equivalent yet illuminating way of extracting a growth law exponent is to define an effective exponent:

$a_{\mathrm{eff}}(t)=\frac{\mathrm{d} \ln R(t)}{\mathrm{d} \ln t}=a-\frac{c a}{R(t)}$

such that a plot of $a_{\text {eff }}$ vs. $1 / R(t)$ should give a straight line whose intersection at the origin is the exponent $a$. This logarithmic derivative is done in practice by using:

$a_{\mathrm{eff}}^{\alpha}(t)=\frac{\log _{\alpha} R(\alpha t)}{\log _{\alpha} R(t)}$

Figure 7 shows the effective exponent when $\alpha=10$, for $R_{\mathrm{a}}$. The extrapolation to the origin gives $a=0.335 \pm 0.010$.

\section{Phase separation in polymer blends}

\subsection{Model and numerical methods}

We consider a mixture of two polymer species, A, B, with chain lengths $N_{\mathrm{A}}=N_{\mathrm{B}}=N$ and subunit size $a_{\mathrm{A}}=a_{\mathrm{B}}=1$. In order to study spinodal decomposition in polymer mixtures, we consider the concentration field $\varphi(r, t)$ of one of the two polymers and choose for $F[\varphi]$ the full Flory-Huggins-de Gennes free energy (in units of $k_{\mathrm{B}} T$ ) given by [22]:

$F[\varphi]=\int \mathrm{d} x\left(f(\varphi(r))+\frac{1}{36 \varphi(1-\varphi)}|\nabla \varphi|^{2}\right)$
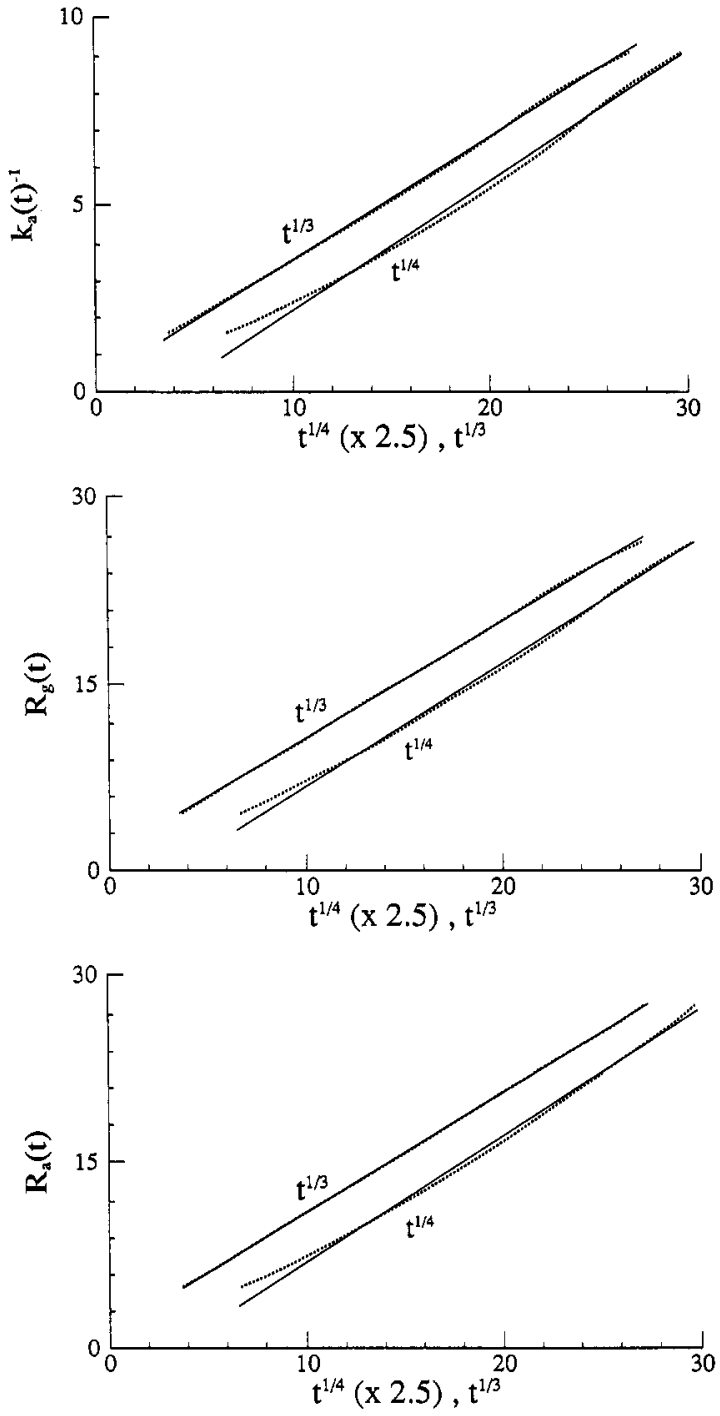

Fig. 6. Domain size as given by the inverse of the first moment of the spherically averaged structure function (see eq. (9)) as a function of $t^{1 / 3}$ and $2.5 t^{1 / 4}$. (b) Same as 6(a) for the domain size $R_{\mathrm{g}}$ defined as the first zero of the spherically averaged pair correlation function. (c) Same as 6(a) for the domain size $R_{\mathrm{a}}$ defined as the first zero of the pair correlation function averaged along the lattice axes.

where,

$f(\varphi(r))=\frac{1}{N}[\varphi \ln \varphi+(1-\varphi) \ln (1-\varphi)]+\chi \varphi(1-\varphi)$

and $\chi$ is the temperature dependent Flory interaction par-

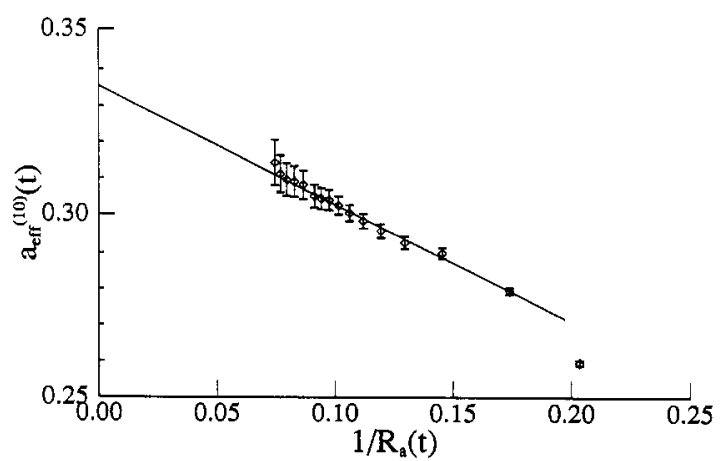

Fig. 7. Effective exponent $a_{\mathrm{eff}}^{(10)}(t)$ as defined in eq. (17) for the measure $R_{\mathrm{a}}$ of the domain size. The ordinate of the straight-line-fit at the origin is $a=$ 0.335 . 
ameter. The resulting equation after substituting expressions (18) and (19) into the general equation (1) is:

$$
\begin{aligned}
\frac{\partial \varphi}{\partial \tau}= & M \nabla^{2}\left[\frac{1}{N} \ln \left(\frac{\varphi}{1-\varphi}\right)-2 \chi \varphi\right. \\
& \left.+\frac{1-2 \varphi}{36 \varphi^{2}(1-\varphi)^{2}}(\nabla \varphi)^{2}-\frac{\nabla^{2}}{18 \varphi(1-\varphi)}\right]+\eta(r, t)
\end{aligned}
$$

In experimental studies $[4,31-34]$, one defines a rescaled wave vector $k$ (in terms of the measured wave-vector $q$ ) and a rescaled time $\tau$ as: $k=q / k_{\mathrm{m}}^{0}$ and $\tau=D_{\mathrm{app}}\left(k_{\mathrm{m}}^{0}\right)^{2} t . k_{\mathrm{m}}^{0}$ and $D_{\mathrm{app}}$ appear in the linear theories and are defined as: $\left(k_{\mathrm{m}}^{0}\right)^{2} \stackrel{\text { app }}{=}$ $\frac{9}{2}\left(\chi-\chi_{\mathrm{s}}\right)$ and $D_{\mathrm{appl}}=2 M\left(\chi=\chi_{\mathrm{s}}\right)$, where $\chi_{\mathrm{s}}=\chi\left(T=T_{\mathrm{c}}\right)=$ $2 / N$. In our numerical studies, we have used the slightly different rescaling: $k_{1}=q\left(\chi-\chi_{\mathrm{s}}\right)^{-1 / 2}$ (correspondingly new space vector $\left.x=r\left(\chi-\chi_{s}\right)^{1 / 2}\right)$ and $\tau_{1}=2 M\left(\chi-\chi_{\mathrm{s}}\right)^{2} t$. The relation between simulation and experimental variables is simply $k_{1}=\sqrt{9 / 2} k$ and $\tau_{1}=2 / 9 \tau$. Equation (20) becomes, in terms of these new reduced variables:

$$
\begin{aligned}
\frac{\partial \varphi}{\partial \tau_{1}}= & \frac{1}{2} \nabla^{2}\left[\frac{\chi_{s}}{2\left(\chi-\chi_{s}\right)} \ln \left(\frac{\varphi}{1-\varphi}\right)-\frac{2 \chi}{\chi-\chi_{s}} \varphi\right. \\
& \left.+\frac{1-2 \varphi}{36 \varphi^{2}(1-\varphi)^{2}}(\nabla \varphi)^{2}-\frac{1}{18 \varphi(1-\varphi)} \nabla^{2} \varphi\right] \\
& +\sqrt{\varepsilon} \xi\left(x, \tau_{1}\right)
\end{aligned}
$$

where the new noise variable $\xi$ satisfies $\left\langle\xi\left(x, \tau_{1}\right) \xi\left(x^{\prime}, \tau_{1}^{\prime}\right)\right\rangle=$ $-\nabla^{2} \delta\left(r-r^{\prime}\right) \delta\left(t-t^{\prime}\right)$ and $\varepsilon=\left(\chi-\chi_{s}\right)^{1 / 2}$. For the polybutadiene system studied in recent experiments [31-32], the critical temperature and the $\chi$ parameter are known [35] and these values are used as the input for the numerical studies. Thus by varying $\chi$ one can faithfully mimic the quench procedure at different final temperatures. We have taken $\chi=$ $0.326 / T-2.3 \times 10^{-4}, T_{\mathrm{c}}=62^{\circ} \mathrm{C}$ and the temperatures 25 , 40,49 and $54.5^{\circ} \mathrm{C}$. With these input parameters, we have numerically integrated eq. (21) on a simple cubic lattice of size $50^{3}$ with periodic boundary conditions and mesh size $\Delta x=1$. We have performed the numerical integration up to $\tau_{1}=500$ using a time step of 0.01 . In order to average over the noise, we have performed 20 runs for each quench temperature. In the following sections, however, we will report our results in terms of the rescaled variable $k$ and $\tau$ used in the experimental studies.

\subsection{Results}

3.2.1. Growth law. In order to study the growth law for the domain size and to examine the issue of scaling, we computed the spherically averaged structure factor $S(k, \tau)$ and the spherically averaged pair correlation function $g(r, \tau)$. We have defined the domain size $R_{\mathrm{g}}(\tau)$ as the coordinate of the first zero of $g(r, \tau)$. We have also computed the location of the maximum of $S(k, \tau), k_{\mathrm{m}}(\tau)$. Our results for $k_{\mathrm{m}}$ are plotted in Fig. 8, for several values of the quench temperature, where we plot the data in a log-log scale. The determination of $k_{\mathrm{m}}$ is difficult since at late times, the maximum of $S(k, \tau)$ is not precisely defined due to the discretization of the Brillouin zone in a finite lattice such as considered here. This explains the small bends in the $k_{\mathrm{m}}$ vs $t$ curves for, say $\tau>500$. Another source of error is the statistical fluctuation of data coming from different runs. For the domain size measures, the statistical errors are of the order of $5 \%$.

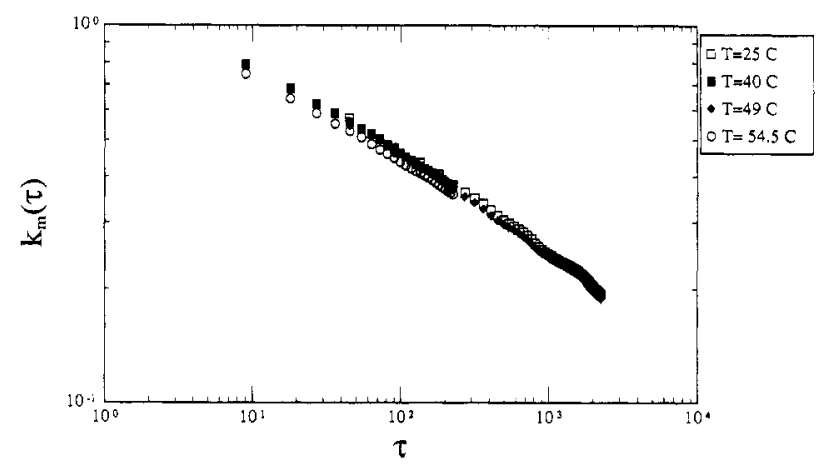

Fig. 8. The maximum of the structure factor, $k_{\mathrm{m}}$ vs. time $\tau$ shown in $\log -\log$ scale for several quench temperatures for the polymer blend studied here (see text). The growth law exponent $(0.28 \pm 0.01)$, computed for $50 \leqslant \tau \leqslant 1000$ is independent of the quench temperature.

In Fig. 8 we see that the growth law exponent is independent of the quench temperature. This result is consistent with the claimed universal scaling of Ref. [33] and [34], but not with that of Ref. [31], where a quench-depth dependent growth law exponent is suggested for early to intermediate stages. The growth law exponent calculated from the slope of these $\log -\log$ plots is given by $0.28 \pm 0.01$. This value of the growth exponent is in excellent agreement with the experimental result for intermediate to "transition" times [32]. We point out that the physical mechanism governing the late time behavior in the model corresponds to the so-called "transition" times in experimental systems, whereas the real late time behavior seen in the experiments is governed by hydrodynamic interactions [36]. We also note that the exponents found from the log-log plots are probably effective exponents, since one expects [26] that the growth law for domain size $R(\tau)$ is given by $R(\tau)=a+b \tau^{n}$. A fit to this expression of the $R_{\mathrm{g}}$ data between $\tau=450$ and $\tau=2250$ yields $n=0.33 \pm 0.01$ for $T=25^{\circ} \mathrm{C}$ and $n=0.33 \pm 0.02$ for $T=49^{\circ} \mathrm{C}$. This value of the exponent suggests that the Lifshitz-Slyozov describes the growth of domains in this time regime.

3.2.2. Scaling. In order to study the scaling hypothesis ansatz eq. (15) for this polymer system, we plot the data for the structure factor in Fig. 9, for several rescaled times $t$ after the quench. If the scaling hypothesis eq. (15) is correct, all the data in Fig. 9 should fall on a single master curve. However, we note that the scaling hypothesis works only at later times, as shown in this figure. Also, although in Fig. 9 the structure factor data are shown only for the quench temperature $25^{\circ} \mathrm{C}$, but the same qualitative picture is found for all the other quench temperatures.

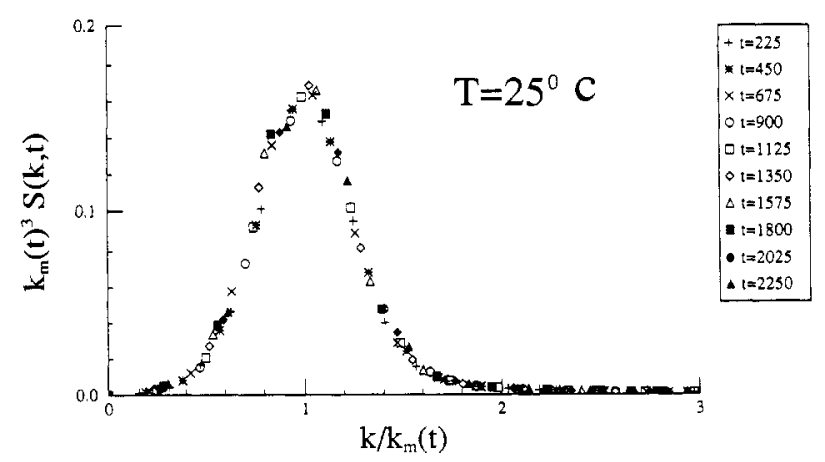

Fig. 9. Plot of the scaling function for the spherically averaged structure factor. Scaling is satisfied only at late times, i.e., $t \geqslant 1350$. The lines are spline fits which serve as a guide to the eye. 


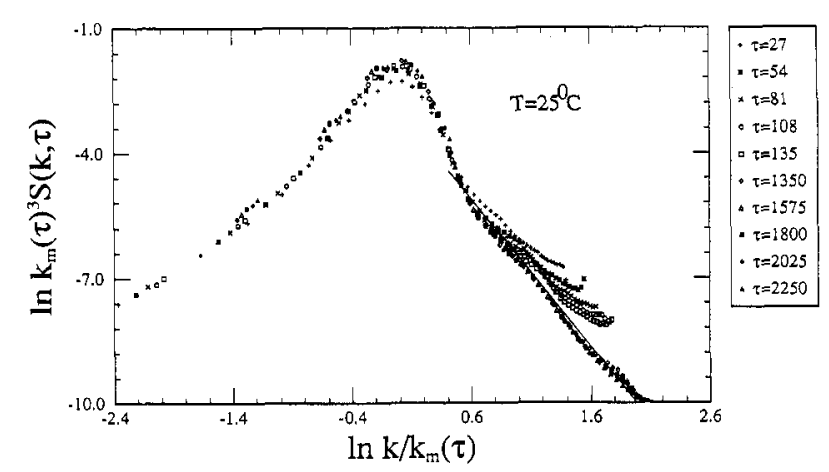

Fig. 10. Plot of the above scaling function in $\log -\log$ scale. The straight line is a fit to the data for large $k$, with a slope of -3.6 .

In order to study the functional form of the scaling curve, we plot the structure factor data in a log-log graph in Fig. 10. It is again clear from this figure that the scaling hypothesis works only at late times when all the curves fall on top of each other. It is interesting to note that the scaling function exhibits a weak shoulder around $k \approx 2 k_{\mathrm{m}}(\tau)$ which has also been observed in the most recent experimental systems [32-33]. The straight line in Fig. 10 is the fit to the data for large $k$-values. We find that for large $k$, the scaling function goes as $k^{-3.6}$, which is close to the expected Porod's Law behavior $\left(k^{-4}\right)$. In recent experimental studies [32-33] it has also been found that for late times the scaling function behaves as $k^{-4}$ for large $k$.

\section{Concluding remarks}

In this paper we have reviewed recent numerical studies of a complicated stochastic nonlinear partial differential equations appropriate for modeling the dynamics of phase separation and pattern formation in binary alloys and polymer blends. An accurate solution of this equation requires the introduction of a space discretization with a very large number of lattice points and a relatively small time step. Also, in order to confirm theoretical ansatz concerning the scaling behavior of the system, it is necessary to solve the equation up to a very late time. Finally, the stochastic nature of the equations requires an average over many realizations. This problem, thus, belongs to the category of those needing an extensive use of the resources (both memory and CPU) of powerful supercomputers. The studies reported here used more than $400 \mathrm{~h}$ of Cray model X-MP48 central-processing unit time. We have focussed mainly on the late time behavior for a reasonably large system. We find that, at sufficiently late times, the scattering intensity and the pair correlation functions are well represented in terms of scaling with a time dependent length. Our analysis of the time dependence of this characteristic length supports a modified Lifshitz-Slyozov law in which the asymptotic growth law exponent is $1 / 3$ for both the binary alloys and polymer blends. We also found that the growth law is independent of final quench temperature for polymer blends. However, a definitive numerical calculation of this controversial exponent would require data spanning much larger times. Also, the exact form for the scaled structure function around the maximum can only be attained in studies involving much larger systems.

Finally, we mention future directions in which we are extending our works. For binary alloy systems a recent simulation by us [37] in the two dimensional version of the model discussed here, has indicated that the scaling functions for both the structure factor and the pair correlation function do not depend appreciably on the volume fraction at least for volume fractions betweeen $20-50 \%$. Although, in numerical simulations it is difficult to probe very large $r$-values in the pair-correlation function (and consequently, very small values of wave vector $k$ in the structure factor), this result is surprising, since analytical [38] and phenomenological [39] theories indicate a much stronger dependence of the scaling functions on the volume fractions. We point out that these analytical theories are valid in three dimensions and for very small volume fractions (typically less than $10 \%$ ). Simulations of off-critical quenches in three dimensions are being carried out to check the validity of these approximate theories.

As mentioned earlier, recent experiments on polymer blends indicate that the growth law for the average domain size depends on the final temperature of quench. We do not see such a temperature dependent growth law in our studies. However, in real polymer mixtures, the Onsager coefficient is wave-vector dependent [22] as well as the $\chi$ parameter depends on composition [40]. We plan to address these questions in future studies.

\section{Acknowledgements}

The study of phase separation in polymer blends discussed here has been carried out in collaboration with Professor M. Muthukumar of the University of Massachusetts, Amherst. We thank Joel Welling of the Pittsburgh Supercomputing Center for numerous help with the graphics used in Fig. 1. This work was supported by NSF grant No. DMR- 8612609 and by an NSF Center. R.T. acknowledges financial support from DGICYT project No. PB-86-0534 (Spain)

\section{References}

1. For a review, see Gunton, J. D., San Miguel, M. and Sahni, P. S., in "Phase Transitions and Critical Phenomena" (Edited by C. Domb and J. L. Lebowitz), Academic, London (1983), vol. 8.

2. Hashimoto, T., in "Dynamics of Ordering Process in Condensed Matter" (Edited by S. Komura and H. Furukawa), Plenum Press, New York (1988).

3. Hashimoto, T, in "Current Topics in Polymer Science", Vol. II, (Edited by R. M. Ottenbrite, L. A. Utracki and S. Inoue), Hanser Publishers, Munich (1987).

4. Hashimoto, T., Phase Transition, 12, 47 (1988).

5. Cahn, J. W. and Hilliard, J. E., J. Chem. Phys. 28, 258 (1958); Cook, H. E., Acta Metall. 18, 297 (1970).

6. Langer, J. S., Ann. Phys. 65, 53 (1971)

7. Langer, J. S., Bar-on, M. and Miller, H. D., Phys. Rev. A11, 1417 (1975).

8. Grant, M., San Miguel, M., Vinals, J. and Gunton, J. D., Phys. Rev. B31, 3027 (1985).

9. Bray, A. J., Phys. Rev. Lett. 62, 2841 (1989); ibid., 63, 818(E) (1989).

10. Mazenko, G. F. and Valls, O. T., Phys. Rev. Lett. 59, 680 (1987); Mazenko, G. F., Valls, O. T. and Zannetti, M., Phys. Rev, B38, 520 (1988).

11. Lifshitz, I. M. and Slyozov, V. V., J. Phys. Chem. Solids, 19, 35 (1961).

12. Lebowitz, J. L., Marro, J. and Kalos, M. H., Acta Metall. 30, 297 (1982) and references therein.

13. Amar, J. G., Sullivan, F. E. and Mountain, R. D., Phys. Rev. B37, 196 (1988)

14. Gunton, J. D., Gawlinski, E. T. and Kaski, K., in "Dynamics of Ordering Processes in Condensed Matter" (Edited by S. Komura and H. Furukawa), Plenum, New York (1988).

15. Gawlinski, E. T., Vinals, J. and Gunton, J. D., Phys. Rev. B39, 7266 (1989).

16. Rogers, T. M., Elder, K. R. and Fesai, R. C., Phys. Rev. B37, 9638 (1988).

17. Oono, Y. and Puri, S., Phys. Rev. Lett. 58, 863 (1987). 
18. Chakrabarti, A. and Gunton, J. D., Phys. Rev. B37, 3798 (1988).

19. Gunton, J. D., Gawlinski, E. T., Chakrabarti, A. and Kaski, K., J. Appl. Cryst. 21, 811 (1988).

20. Toral, R.. Chakrabarti, A. and Gunton. J. D., Phys. Rev. Lett. 60, 2311 (1988).

21. Chakrabarti, A., Toral, R. and Gunton, J. D., Phys. Rev. B39, 4386 (1989).

22. de Gennes, P. G., J. Chem. Phys. 72, 4756 (1980); Pincus, P., J. Chem. Phys. 75, 1996 (1981); Binder, K., J. Chem. Phys. 79, 6387 (1983).

23. Baumgartner, A. and Heerman, D. W., Polymer, 27, 1777 (1986).

24. Sariban, A. and Binder, K., Polym. Commun. 30, 205 (1989).

25. Chakrabarti, A., Toral, R., Gunton, J. D. and Muthukumar, M., Submitted to Phys. Rev. Lett.

26. Huse. D. A., Phys. Rev. B34, 7845 (1986).

27. Oki, K., Sagana, H. and Eguchi, T., J. Phys. (Paris) C7, 414 (1977),

28. Binder, K., Z. Phys. B43, 119 (1981).

29. Furukawa. H., Physica 123A, 497 (1984) and references therein.
30. Mazenko, G. F., University of Chicago preprint (1989).

31. Wiltzius, P., Bates, F. S. and Heffner, W. R., Phys. Rev. Lett. 60, 1538 (1988).

32. Bates, F. S. and Wiltzius, P., J. Chem. Phys. 91, 3258 (1989).

33. Hashimoto, T., Takenaka, M. and Izumitani, T., Polym. Commun. 30, 45 (1989).

34. Snyder, H. L. and Meakin, P., J. Chem. Phys. 79, 5588 (1983).

35. Bates, F. S., Wignall, G. D. and Koehler, W. C., Phys. Rev. Lett. 55. 2425 (1985).

36. Siggia, E. D., Phys. Rev. A20, 595 (1979).

37. Toral, R., Chakrabarti, A. and Gunton. J. D., Phys. Rev, B39, 901 (1989)

38. Tokuyama, M., Enomoto, Y. and Kawasaki. K.. Physica A143, 183 (1987).

39. Fratzl, P. and Lebowitz, J. L., to appear in Acta Metallurgica (1989).

40. Bates, F. S., Muthukumar, M., Wignall. G. D.. Fetters, L. J.. J. Chem. Phys. 89, 535 (1989). 